\title{
RESEARCH
}

Open Access

\section{Blood biomarkers for stroke: the role of thioredoxin in diagnosis and prognosis of acute ischemic stroke}

\author{
Mohammed I. Oraby ${ }^{1 *}$ (iD and Radwa A. Rabie ${ }^{2}$
}

\begin{abstract}
Background: Oxidative stress plays a crucial role in the pathophysiology of acute ischemic stroke. Thioredoxin exists and released from cells during inflammation and oxidative stress and was recognized as an oxidative-stress marker.

Objective: The objective of this study was to assess the role of thioredoxin as an oxidative stress biomarker in diagnosis and prognosis of acute ischemic stroke in a sample of patients recruited from Beni-Suef Governorate, north Upper Egypt.
\end{abstract}

Methods: A case control study included 100 subjects; 50 patients with first-ever acute ischemic stroke presented within $24 \mathrm{~h}$ from the onset and 50 healthy volunteers as a control. Clinical, functional, and radiological evaluation was done for the patients, and all patients and control were subjected to routine laboratory tests and assessment of serum level of thioredoxin by solid-phase sandwich enzyme-linked immunosorbent assay.

Results: Thioredoxin was significantly higher in acute stroke patients compared to control group ( $p$ value $=0.001$ ). Thioredoxin level was significantly higher in hypertensive patients ( $p$ value $=0.007$ ), patients who had carotid stenosis $\geq 50 \%$ ( $p$ value $=0.001$ ), patients with poor outcome ( $p$ value $=0.009)$, and in patients with cardio-embolic stroke $(p$ value $=0.001)$.

Significant positive correlation was found between thioredoxin level and volume of infarction $(r=0.501$ and $p=$ $0.001)$, stroke severity at presentation $(r=0.503$ and $p=0.021)$ and clinical outcome after 3 months $(r=0.551$ and $p$ value $=0.001$.

Conclusion: Thioredoxin as a marker of oxidative stress can be used as a new diagnostic and prognostic blood biomarker for stroke.

Keywords: Stroke, Thioredoxin, Oxidative stress, NIHSS, mRS

\section{Introduction}

Acute ischemic stroke carries an enormous health burden, since it is rated to be one of the main causes of disability and even mortality [1]. Developing valid biomarkers for accurate diagnosis and assessment of prognosis of acute ischemic stroke is a key goal.

In the last few years, there has been a considerable evidence to suggest that oxidative stress associated with excessive production of reactive oxygen species (ROS) is a primary and fundamental mechanism of ischemic

\footnotetext{
* Correspondence: Drm_Oraby@med.bsu.edu.eg; Drm_Oraby@hotmail.com 'Department of Neurology, Beni-Suef University, Beni-Suef 62511, Egypt Full list of author information is available at the end of the article
}

brain damage in stroke and reperfusion injury ensuing stroke [2].

Compared to other organs, the brain is more vulnerable to oxidative damage due to various factors that enhance the formation of reactive species: high oxygen consumption, high fat content of unsaturated fatty acids, and high iron levels in some brain regions. At the same time, the brain has low levels and low activities of some antioxidant enzymes as catalase, superoxide dismutase (SOD), and glutathione peroxidase [3].

Thioredoxin system consists of thioredoxin, thioredoxin reductase, and NADPH. Thioredoxin is a ubiquitous small protein $(12 \mathrm{KDa})$ which has a redox-active 
disulfide/dithiol site. Through thiol redox control, thioredoxin regulates several cellular and biological functions. Also, thioredoxin gene has a novel cis-regulatory protein in its promoter region responsive to oxidative stress and can be strongly induced by oxidative stress such as ischemic injury, various oxidative agents, and irradiation $[4,5]$. Thioredoxin reflects both oxidative stress and inflammation and was recognized as an oxidative-stress marker [6].

Studies which assessed thioredoxin as a marker of oxidative-stress in acute ischemic stroke are few with conflict results. The aim of this study was to evaluate the potential role of serum thioredoxin level as a serological marker in diagnosis of acute ischemic stroke and to clarify the relation between serum thioredoxin level and the clinical outcome of acute ischemic stroke.

\section{Methods}

This study is a case control study conducted in neurology department, Beni-Suef University during the period from June to December 2018 and included 100 subjects; 50 patients with first-ever acute ischemic stroke presented within $24 \mathrm{~h}$ from the onset and 50 healthy volunteers who do not have a family history of stroke matched in age and sex to stroke patients as a control.

Patients with the following conditions were excluded from the study: hemorrhagic stroke, venous infarction, autoimmune diseases, acute or chronic infection, and surgery or trauma during the previous three months. A written informed consent was obtained from each participant in this study or from one of his family members, and the study was approved by the authorized ethical committee in Faculty of Medicine, Beni-Suef University (FWA00015574 in 23/6/2018).

All patients included in this study were subjected to the following battery of assessment at presentation;

Clinical assessment: patients were submitted to clinical evaluation including detailed history taking, general medical examination including a cardiological assessment, and neurovascular examination according to the cerebrovascular stroke assessment sheet of Neurology Department, Beni Suef University. Disease severity at presentation was assessed using the National Institute of Health Stroke Scale (NIHSS) [7]. Stroke severity assessed by NIHSS was categorized as mild (score: $1-5$ ), moderate (score: $6-13$ ), or severe (score: $\geq 14$ ) [8]. After three months, the patient group was subjected to assessment of functional outcome using Modified Rankin Scale (mRS) and categorized as good (mRS 0 to 2; independent) or poor (mRS 3 to 6; dependent or dead) [9].

Laboratory assessment: patients and control groups were subjected to the following laboratory investigations; routine investigations (fasting and post-prandial blood sugar, complete blood count, and ESR, serum electrolytes, serum uric acid, kidney and liver function tests, and lipid profile).

Patients and control groups were also subjected to evaluation of Serum Thioredoxin level within the first 24 $\mathrm{h}$ of admission. Serum thioredoxin levels were measured by enzyme-linked immunosorbent assays (ELISA) using Stat Fax 2100 Microplate Reader, USA, in Clinical and Chemical Pathology Department, Beni-Suef University hospital. Blood samples were prospectively drawn from the cubital vein on the first morning after the admission in the patients or on the morning of inspection day in controls. Samples were immediately centrifuged and stored at $-80{ }^{\circ} \mathrm{C}$ before batch testing. The serum level of TRX was measured using a solid-phase sandwich enzyme-linked immunosorbent assay. The lower detection limit was $0.45 \mathrm{ng} / \mathrm{mL}$, and the line range was $0.45-$ $100 \mathrm{ng} / \mathrm{mL}$.

Radiological assessment including brain computed tomography $(\mathrm{CT})$ using Toshiba multislice 16 computed tomography, Japan and MRI using Philips Achieva 1.5 tesla machine, Netherland, was carried out for all patients in Radiology Department, Beni-Suef University Hospital. Hemorrhagic stroke, old stroke cases, and venous infarction were excluded. Infarction volume in DWI was measured using the following formula: $A \times B \times C / 2$, where $\mathrm{A}$ is the largest diameter, $\mathrm{B}$ is the perpendicular diameter of the ischemic lesion, as measured, and $C$ is the sum of the thicknesses of the slices where the lesion was visible [10]. Stroke subtype was classified according to TOAST (Trial of Org 10172 in Acute Stroke Treatment) criteria [11], which distinguished small-artery occlusion, large-artery arteriosclerosis, cardioembolism, other causative factors, and undetermined causative factor.

Electrocardiography (ECG): using standard twelve leads electrocardiogram in Cardiology Department, Beni-Suef University Hospital.

Echocardiography: vivid S5 machine, China, transthoracic echo-Doppler study using $2 \mathrm{D}$ and $\mathrm{M}$ mode was done by trained cardiologist in cardiology department, Beni-Suef University Hospital.

Carotid duplex: Toshiba machine carotid duplex, Japan, using B mode and Doppler flow was done by radiologist in radiology department in Beni-Suef University Hospital.

\section{Statistical analysis}

Data was coded and entered using Microsoft Office Excel 2010. Statistical analysis was done using IBM SPSS version 24 (IBM Corporation, USA, Armonk, NewYork, 2016). Frequencies (number) and relative frequencies (percent) were used to summarize qualitative variables while mean and standard deviations were used for quantitative variables. Comparisons between groups were 
done using chi-square test, independent sample $t$ test and one-way ANOVA test. Receiver operating characteristic (ROC) curves were used to estimate the optimal cut-off thioredoxin levels to predict poor outcome after acute ischemic stroke and to predict cardio-embolic strokes. Pearson correlation was used to test for possible correlations between quantitative variables. Binary logistic regression (backward method) was used to determine predictors of bad outcome of stroke. Variables entered in the equation were Thioredoxin level, sex, diabetes, hypertension, smoking, and type of stroke. $P$ value less than or equal to 0.05 was considered significant.

\section{Results}

This study included 50 patients with acute ischemic stroke admitted within $24 \mathrm{~h}$ from the onset of symptoms and 50 healthy volunteers as a control group. Age of the cases ranged from 29 to 78 years with a mean of $55.94 \pm$ 12.84, while the age of control ranged from 28 to 70 years with a mean of $49.38 \pm 13.43$. Patients were 24 males (48\%) and 26 females (52\%), while control were 27 males (54\%) and 23 females (46\%).

The results of clinical assessment scales which were done for stroke patient at admission and after 3 months are shown in Table 1. The mean of NIHSS score at admission was $10.6 \pm 5.47$ (range 3-19) and for mRS after 3 months was $1.23 \pm 2.05$ (range 1-4).

According to the resulting mRS score after 3 months, $33(66 \%)$ patients were classified as having good outcome (grades 1 and 2) and 17 (34\%) patients were with poor outcome (grades 3 and 4).

Regarding the volume of infarction, 31(62\%) patients had infarction volume from 0.5 to $5 \mathrm{ml}, 9$ (18\%) patients had infarction volume from 5 to $10 \mathrm{ml}$, and $10(20 \%)$ patients had infarction volume of more than $10 \mathrm{ml}$, with a mean of $7.25 \pm 10.75$.

Table 1 Results of NIHSS and mRS in 50 acute ischemic stroke patients.

\begin{tabular}{llll}
\hline Scale & Score & Number (50) & Percent (\%) \\
\hline NIHSS on admission & Mild (1-5) & 20 & $40 \%$ \\
& Moderate (6-13) & 17 & $34 \%$ \\
& Severe ( $\geq 14)$ & 13 & $26 \%$ \\
mRS after 3 months & 0 & 0 & $0 \%$ \\
& 1 & 20 & $40 \%$ \\
& 2 & 13 & $26 \%$ \\
& 3 & 10 & $20 \%$ \\
& 4 & 7 & $14 \%$ \\
& 5 & 0 & $0 \%$ \\
\hline
\end{tabular}

NIHSS National Institute of Health Stroke Scale, mRS modified Rankin Scale
According to TOAST classification, 14 patients $(28 \%)$ had small artery stroke, 22 patients (44\%) had large artery stroke, and $14(28 \%)$ patients had cardio embolic stroke.

Thioredoxin level in acute stroke patients and control group was shown in Table 2. Thioredoxin was significantly higher in acute stroke patients compared to control group $(p=0.001)$.

On comparing between patients with good and poor clinical outcome regarding thioredoxin level, thioredoxin was significantly higher in patients with poor outcome than in patients with good outcome $(p$ value $=0.009)$ (Table 2).

Table 3 shows the relation between some of the most common modifiable and non-modifiable risk factors of stroke and thioredoxin level at presentation. A statistically significant high levels of thioredoxin were shown in hypertensive patients with acute stroke and those having $50 \%$ or more carotid stenosis, in comparison to others with other risks ( $p=0.007, p=0.001)$, respectively.

Using receiver operator characteristic analysis (ROC) curve, the optimal cutoff value of thioredoxin that predicted poor outcome after acute ischemic stroke was $21.9 \mathrm{ng} / \mathrm{ml}$, with sensitivity of $88 \%$ and specificity of $64 \%$ (the areas under the curve $0.75,95 \%$ confidence interval 0.608 to $0.891, P$ value 0.002 ) (Fig. 1).

Also, thioredoxin levels in patients with cardioembolic stroke was significantly higher than patients with other ischemic stroke subtypes $(p$ value $=0.001)$ (Table 4).

Using ROC curve, the optimal cutoff value of thioredoxin that predicted cardio-embolic stroke was 25.165 $\mathrm{ng} / \mathrm{ml}$, with sensitivity of $85.7 \%$ and specificity of $66.7 \%$ (the areas under the curve $0.85,95 \%$ confidence interval 0.732 to $0.967, P$ value $<0.001$ ) (Fig. 2).

There was a statistically significant moderate positive correlation between thioredoxin level and volume of infarction measured by MRI $(r=0.501$ and $p=0.001)$, stroke severity at presentation measured by NIHSS $(r=$ 0.503 and $p=0.021$ ) and clinical outcome after 3 months measured by $\mathrm{mRS}(r=0.551$ and $p$ value $=$ 0.001) Table 5.

Binary logistic regression (backward method) was used to determine predictors of bad outcome of stroke. Variables entered in the equation were thioredoxin level, sex, diabetes, hypertension, smoking, and type of stroke. Among studied stroke patients, higher thioredoxin level (OR and $P$ value of 1.139 and 0.011 ) and being hypertensive (OR and $P$ value of 3.232 and 0.039 ) were found to be significant predictors of bad outcome of stroke. Hosmer-Lemeshow (H-L) test that yielded a $\chi^{2}$ (8) of 10.196 and was insignificant $(p>0.05)$, suggesting that the model was fit to the data well (Table 6). 
Table 2 Thioredoxin level in different clinical situations

\begin{tabular}{llll}
\hline & Number & $\begin{array}{l}\text { Thioredoxin level (ng/ml) } \\
\text { Mean } \pm \text { SD }\end{array}$ & $P$ value \\
\hline Control group & & $10.00 \pm 2.99$ & 0.001 \\
Stroke patients & 50 & $24.73 \pm 7.13$ & $23.46 \pm 8.38$ \\
Stroke patients with good outcome & 50 & $29.78 \pm 7.91$ & 0.009 \\
Stroke patients with poor outcome & $33 / 50$ & &
\end{tabular}

$P$ value $<0.05$ (significant)

\section{Discussion}

The early hours after an acute stroke are crucial; it is the most useful time for effective management. With the rising burden of stroke and marked heterogeneity in stroke manifestation and outcome, it is necessary to find accurate, reliable, and simple predictors of functional recovery [12].

Accumulating evidences suggest that oxidative stress and inflammation play a crucial role in the pathophysiology of acute ischemic stroke [13]. The accurate measurement of oxidative stress in stroke would be of extreme value for a better understanding of its pathophysiology and for identifying patients' subgroups that might benefit from receiving targeted therapeutic intervention [14]. Blood biomarkers of oxidative stress can be used as early indicators of stroke. Currently, an accurate and valid biomarker is still under investigations [15].

Several studies reported a close relation between increased expression of thioredoxin and cell damage due to oxidative stress. Thioredoxin system is up regulated in postmortem examination of Alzheimer disease in human and after experimental middle cerebral artery occlusion in rats [16]

We found that thioredoxin level was significantly higher in acute stroke patients compared to control group $(p=0.001)$. This finding is in accordance with the results of most of the studies which assessed thioredoxin as a marker of oxidative stress in acute ischemic stroke $[15,17,18]$.

Similarly, Ozkul, and colleagues [19] reported that oxidative stress plays a crucial role in the pathogenesis of acute ischemic stroke, and subsequent oxidative damage caused by free radical formation may be factors in stroke severity, while Carbonell and colleagues [20] found that following ischemic stroke, iron-dependent oxidative stress in the penumbra can enhance necrosis and further neurological deterioration.

On the other hand, Al-Hussain and colleagues [21] in their study which included 45 acute ischemic stroke patients found no statistically significant difference between serum level of thioredoxin in their acute ischemic stroke patients and control group. The mean age of

Table 3 Relation between risk factors of stroke and thioredoxin level at presentation in 50 acute stroke patients

\begin{tabular}{|c|c|c|c|c|c|c|}
\hline \multicolumn{4}{|l|}{ Risk factor } & \multicolumn{3}{|c|}{ Thioredoxin level at presentation } \\
\hline & & Number & Percent & Mean & SD & $P$ value \\
\hline \multirow[t]{2}{*}{ Age } & $\leq 45$ & 14 & $28 \%$ & 26.81 & 8.85 & 0.202 \\
\hline & $>45$ & 36 & $72 \%$ & 23.93 & 6.30 & \\
\hline \multirow[t]{2}{*}{ Sex } & Males & 24 & $48 \%$ & 24.39 & 6.03 & 0.750 \\
\hline & Females & 26 & $52 \%$ & 25.05 & 8.12 & \\
\hline \multirow[t]{2}{*}{ Smoking } & Yes & 6 & $12 \%$ & 22.70 & 5.99 & 0.463 \\
\hline & No & 44 & $88 \%$ & 25.01 & 7.29 & \\
\hline \multirow[t]{2}{*}{ Diabetes Mellitus } & Yes & 12 & $24 \%$ & 21.71 & 6.04 & 0.093 \\
\hline & No & 38 & $76 \%$ & 25.69 & 7.25 & \\
\hline \multirow[t]{2}{*}{ Hypertension } & Yes & 16 & $32 \%$ & 26.29 & 7.63 & $0.007^{*}$ \\
\hline & No & 34 & $68 \%$ & 21.42 & 4.58 & \\
\hline \multirow[t]{2}{*}{ Dyslipidemia } & Yes & 12 & $24 \%$ & 23.60 & 5.87 & 0.430 \\
\hline & No & 38 & $76 \%$ & 25.71 & 6.99 & \\
\hline \multirow[t]{2}{*}{ Carotid stenosis } & $<50 \%$ & 11 & $22 \%$ & 21.75 & 5.23 & $0.001 *$ \\
\hline & $\geq 50 \%$ & 39 & $78 \%$ & 31.07 & 6.56 & \\
\hline
\end{tabular}

${ }^{*} P$ value $<0.05$ (significant) 


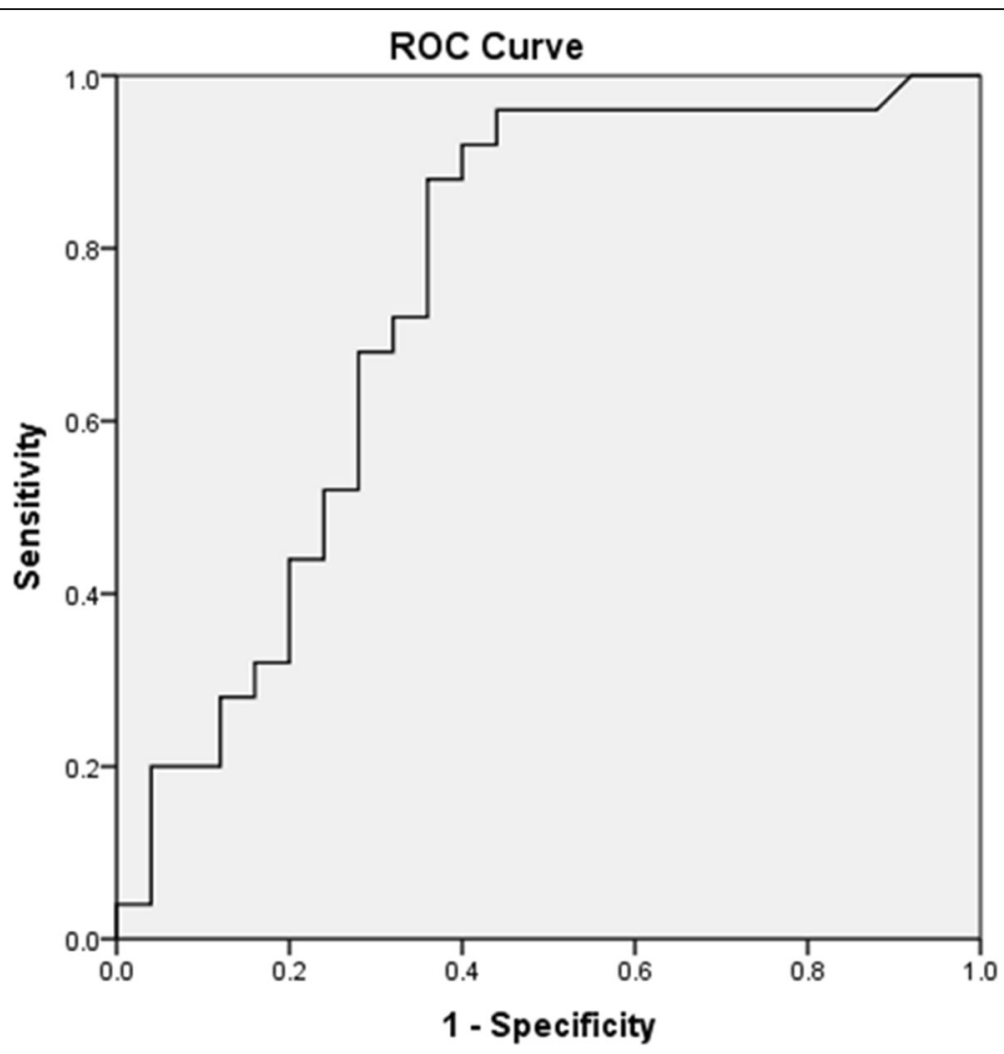

Fig. 1 ROC curve for prediction of poor outcome after acute ischemic stroke using thioredoxin level on admission

patients included in this study was $40 \pm 16$ which is significantly younger than our study population (55.94 \pm 12.84). Also, they did not include in their study any data about stroke severity at presentation, type of stroke or volume of infarction, underlying risk factors, or stroke outcome.

We studied the relation between some of the most common modifiable and non-modifiable risk factors of stroke (age, sex, smoking, diabetes mellitus, hypertension, dyslipidemia, and carotid stenosis $\geq 50 \%$ ) and thioredoxin level at presentation. Thioredoxin level was significantly higher in hypertensive patients and in patients who had carotid stenosis, $\geq 50 \%$. Whereas there was no significant relation between thioredoxin level and age, sex, smoking, diabetes mellitus, or dyslipidemia.

Hypertension is considered as one of the most important risk factor for cerebrovascular diseases [22].

Table 4 Comparison between different acute ischemic stroke subtypes regarding the mean thioredoxin level

\begin{tabular}{llll}
\hline & $\begin{array}{l}\text { Number } \\
(\%)\end{array}$ & $\begin{array}{l}\text { Thioredoxin level } \\
\text { Mean } \pm \text { SD }\end{array}$ & $P$ value \\
\hline Small artery stroke & $14(28 \%)$ & $16.65 \pm 2.00$ & $0.001^{*}$ \\
Large artery stroke & $22(44 \%)$ & $25.58 \pm 3.55$ & \\
Cardioembolic stroke & $14(28 \%)$ & $31.49 \pm 6.82$ & \\
\hline
\end{tabular}

${ }^{*} P$ value $<0.05$ (significant)
Oxidative stress has gained more attention as one of the primary mechanisms responsible for the generation of hypertension. Reactive oxygen species have an important role to play in the homeostasis of the vessel wall. In arteries isolated from hypertensive rats and humans, antioxidant bioactivity is reduced, redoxdependent signaling is enhanced, and ROS production is increased [23].

In addition, the mechanical stimulation on the vascular wall which increases with hypertension may increase ROS production. ROS-induced vasoconstriction results from reduced nitric oxide (NO) levels and increased intracellular calcium concentration and as a result contributing to the pathogenesis of hypertension [24].

Carotid artery atherosclerotic disease accounts for a considerable percentage of all strokes [25]. Oxidative stress plays a crucial role in the pathogenesis of atherosclerotic vascular disease [26].

Studies have demonstrated that oxidative stress is a fundamental feature of the atherogenesis [27]. The redox status occurs when imbalance happens between antioxidant capacity and activity species including reactive nitrogen and halogen species, ROS, non-radical as well as free radical species [28]. This imbalance leads to cell injury by directly oxidizing cellular lipid, protein, and DNA or by initiating cell death signaling pathways [29]. 


\section{ROC Curve}

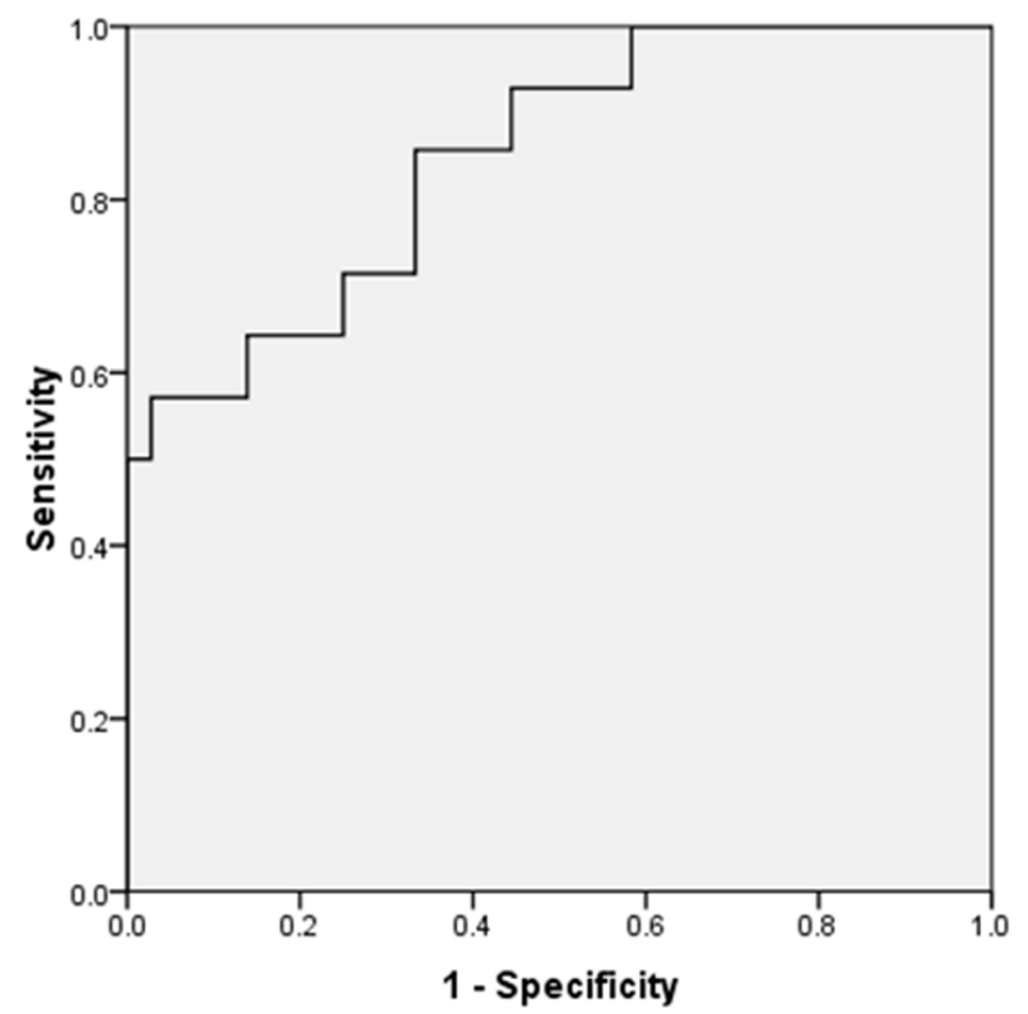

Fig. 2 ROC curve for prediction of cardio-embolic stroke using thioredoxin level on admission

Moreover, other studies demonstrated that ROS have been implicated in matrix metalloproteinases activation, endothelial dysfunction, and migration of vascular smooth muscle cell [30]. Also, ROS can promote vascular disease by reduction of NO bioavailability resulting in lower antiapoptotic and vasodilator action of NO [31].

In this study, there was a statistically significant positive correlation between serum level of thioredoxin and stroke severity at presentation measured by NIHSS ( $r=$ 0.503 and $p=0.021$ )

This is in accordance with Qi and colleagues [15] and $\mathrm{Wu}$ and colleagues [16] studies which also found a significant positive association between serum thioredoxin levels and NIHSS scores.

In contrast, Tieer and colleagues [18] found negative correlation between NIHSS score at admission and serum thioredoxin levels in their cardioembolic stroke patients.

Also, we found a statistically significant positive correlation between thioredoxin level and volume of infarction measured by MRI ( $r=0.551$ and $p=0.001)$. Supporting our results, Nanetti and colleagues [32] found that changes in the generation of free radicals and as a result oxidative stress may play a role in the pathogenesis and extent of acute ischemic lesions.

Table 5 Correlation between thioredoxin level, radiological, and clinical parameters

\begin{tabular}{lll}
\hline & Thioredoxin level & Probability value $(P$ value $)$ \\
\cline { 2 - 3 } Volume of infarction & Correlation coefficient $(r)$ & 0.001 \\
Stroke severity at presentation (measured by NIHSS) & 0.501 & 0.021 \\
Clinical outcome after 3 months (measured by mRS) & 0.503 & 0.001 \\
\hline
\end{tabular}

$P$ value $<0.05$ (significant) 
Table 6 Logistic regression analysis for predictors of poor outcome among studied stroke patients

\begin{tabular}{|c|c|c|c|c|c|c|c|c|}
\hline \multirow[t]{2}{*}{ Predictor } & \multirow[t]{2}{*}{ B } & \multirow[t]{2}{*}{ SE } & \multirow{2}{*}{$\begin{array}{l}\text { Wald } \\
\left(x^{2}\right)\end{array}$} & \multirow[t]{2}{*}{$d f$} & \multirow{2}{*}{$\begin{array}{l}P \\
\text { value }\end{array}$} & \multirow[t]{2}{*}{ OR } & \multicolumn{2}{|c|}{$95 \% \mathrm{Cl}$ for OR } \\
\hline & & & & & & & Lower & Upper \\
\hline Constant (a) & -4.503 & 1.505 & 8.958 & 1 & 0.003 & NA & NA & NA \\
\hline Thioredoxin level & 0.130 & 0.051 & 6.482 & 1 & 0.011 & 1.139 & 1.030 & 1.259 \\
\hline Hypertension ( $1=$ yes, $0=$ no) & 1.173 & 0.689 & 2.898 & 1 & 0.039 & 3.232 & 0.837 & 12.476 \\
\hline
\end{tabular}

$B$ regression coefficient, $S E$ standard error, $X^{2}$ chi square test statistic, $d f$ degree of freedom, $O R$ odds ratio, $C l$ confidence interval

This is in accordance with Qi and colleagues' [15] and Wu and colleagues' [17] while Tieer and colleagues [18] found serum thioredoxin levels in patients with small infarction were not significantly different from those in the large infarction volume group.

Regardless of the mechanisms responsible for ischemic stroke, a sequence of events through different pathways increases the production of free radicals. During ischemia, as a result of deficiency of oxygen and glucose substrates, ATP cannot be produced which leads to inactivation of ATP-dependent ion pumps, membranes depolarization, and disturbance in trans membrane ion gradients. As a result, calcium is translocated from extracellular to intracellular spaces, activating cellular lipases and proteases and the subsequent cerebral tissue breakdown [33].

We found that thioredoxin was significantly higher in patients with poor outcome compared to patients with good outcome. Using ROC curve, the best cutoff limit of thioredoxin levels early after admission (in the first $24 \mathrm{~h}$ of stroke) in predicting poor outcome was $21.89 \mathrm{ng} / \mathrm{ml}$ ( $88 \%$ sensitivity and $64 \%$ specificity). Higher thioredoxin level was found to be significant predictors of bad outcome of stroke (OR and $P$ value of 1.139 and 0.011 ) using binary logistic regression. We also found a statistically positive correlation between thioredoxin level and clinical outcome after 3 months measured by mRS.

This finding is similar to Qi and colleagues study [15] which included 312 patients and found that serum thioredoxin levels were higher compared with those in patients with a favorable outcome, while their optimal cutoff value of serum thioredoxin levels as an indicator for prognosis of functional outcome based on the ROC curve was slightly lower than our results, $20.0 \mathrm{ng} / \mathrm{ml}$ ( $82.8 \%$ sensitivity and $78.9 \%$ specificity).

Also, Wu and colleagues [17] found that serum thioredoxin levels were higher in patients with unfavorable outcome compared with those in patients with a favorable outcome.

In contrast, Tieer and colleagues [18] in their study which included 198 acute ischemic stroke patients found that serum thioredoxin levels in the favorable outcome group were significantly higher than in the unfavorable outcome group.

The conflict between the results of our study supported by the results of Qi and colleagues [15] and Wu and colleagues [17] on one hand and the results of Tieer and colleagues [18] on the other hand regarding the relation between thioredoxin level and stroke severity at presentation, volume of infarction, and functional out come after 3 months may be explained by the differences in timing of sampling (onset of symptom within $24 \mathrm{~h}$ versus $72 \mathrm{~h}$ ). The median NIHSS score in our study was 10.6 and in Qi and colleagues' and Wu and colleagues' studies was 9 while in Tieer study of the population, the median score was only 4 points. Also, there are different indicators of functional outcome (modified Rankin Scale versus Barthel Index). Another possible cause may be the heterogeneity of patients as $28 \%$ of strokes in our study was cardioembolic and in Qi and colleagues' and Wu and colleagues' studies was 31.4\% while in Tieer study was only $18.7 \%$.

In this study, thioredoxin levels in patients with cardio-embolic stroke was significantly higher than patients with other ischemic stroke subtypes ( $p$ value $<0.001$ ). Using ROC curve, the best cutoff limit of thioredoxin levels done to patients early after admission in the first $24 \mathrm{~h}$ of stroke predicting cardioembolic stroke was $25.165 \mathrm{ng} / \mathrm{ml}(85.7 \%$ sensitivity and $66.7 \%$ specificity).

Meanwhile, Qi and colleagues [15] and Wu and colleagues [17] found that the median thioredoxin levels were significantly greater for large-vessel occlusive than for the other stroke subtype groups $(p$ value $=0.0001)$. On the other hand, Tieer and colleagues [18] found no relation between thioredoxin level and different stroke syndromes.

Limitations of this study include the relatively small number of patients due to the limitation of resources and financial issues, and we measured thioredoxin level in serum, not in cerebral spinal fluid. Whether the peripheral thioredoxin levels reflect similar changes in the central nervous system remains uncertain.

Thioredoxin is a single oxidative stress parameter provides only one-sided and partial insights into neuronal dysfunction and death mediated by free radical

\section{Conclusions}

Serum thioredoxin level as an indicator for oxidative stress was higher in acute stroke patients compared to healthy control and positively correlate with stroke severity at presentation measured by NIHSS and functional outcome measured by mRS after 3 months and 
can be used as a new diagnostic and prognostic blood biomarker for stroke. Further studies should be conducted to clarify the effect of different therapeutic lines used in management of acute ischemic stroke on thioredoxin level and if thioredoxin can be used as an indicator for the response to such therapeutic lines.

\section{Abbreviations}

ROS: Reactive oxygen species; SOD: Superoxide dismutase; NIHSS: National Institute of Health Stroke Scale; ELISA: Enzyme-linked immunosorbent assays; mRS: Modified Rankin Scale; TOAST: Trial of Org 10172 in Acute Stroke Treatment; ROC: Receiver Operator Characteristic; NO: Nitric oxide

\section{Acknowledgements}

Not applicable

\section{Authors' contributions}

$\mathrm{MO}$ participated in the study design, collection of data, sequence alignment, and analysis of data and helped to draft manuscript. RR participated in the study design, performed the laboratory work, and helped to draft manuscript. All authors read and approved the final manuscript.

\section{Funding}

Authors did not receive any funding for this work.

\section{Availability of data and materials}

The datasets used and/or analyzed during the current study are available from the corresponding author on reasonable request with the permission of Faculty of Medicine, Beni-Suef University, Egypt.

\section{Ethics approval and consent to participate}

A written informed consent was obtained from each participant in this study, and the study was approved by the authorized ethical committee in Faculty of medicine, Beni-Suef University (FWA00015574) in 23-6-2018.

\section{Consent for publication}

Not applicable

\section{Competing interests}

The authors declare that they have no competing interests.

\section{Author details}

'Department of Neurology, Beni-Suef University, Beni-Suef 62511, Egypt.

${ }^{2}$ Clinical Pathology Department, Beni-Suef University, Beni-Suef, Egypt.

\section{Received: 9 December 2018 Accepted: 4 October 2019}

Published online: 31 December 2019

\section{References}

1. Murray CJ, Lopez AD. Measuring the global burden of disease. N Engl J Med. 2013;369:448-57

2. Allen $\mathrm{CL}$, Bayraktutan U. Oxidative stress and its role in the pathogenesis of ischaemic stroke. Int. J. Stroke. 2009:4(6):461-70.

3. Dringen R, Gutterer JM, Hirrlinger J. Glutathione metabolism in brain: metabolic interaction between astrocytes and neurons in the defense against reactive oxygen species. European Journal of Biochemistry. 2000;267(16):4912-6.

4. Maulik N, Das DK. Emerging potential of thioredoxin and thioredoxin interacting proteins in various disease conditions. Biochim Biophys Acta Gen 2008 Subj. 1780;11:1368-82.

5. Holmgren A. Thioredoxin. Annu Rev Biochem. 1985;54:237-71.

6. Burke-Gaffney A, Callister ME, Nakamura $H$. Thioredoxin: friend or foe in human disease? Trends Pharmacol Sci. 2005:26:398-404.

7. NIH Stroke Scale. National Institute of Neurological Disorder website. https:// www.ninds.nih.gov/sites/default/files/NIH_Stroke_Scale.pdf. Accessed May 2018

8. Chlegel D, Kolb SJ, Luciano JM, Tovar JM, Cucchiara BL, Liebeskind DS, Kasner SE. Utility of the NIH Stroke Scale as a predictor of hospital disposition. Stroke. 2003;34:134-7.

9. Bonita R, Beaglehole R. Modification of Rankin Scale: Recovery of motor function after stroke. Stroke. 1988;19(12):1497-500.
10. Park YW, Koh EJ, Choi HY. Correlation between serum D-dimer level and volume in acute ischemic stroke. J Korean Neurosurg Soc. 2011;50:89-94.

11. Adam HP, Bendixen BH, Krapple LJ, Biller J, Love BB, Gordon DL, et al. Classification of acute ischemic stroke. Difinitions for use in a multicenter clinical trial. Stroke. 1993;24:35-41.

12. Hashem S, Helmy S, El-Fayomy N, Oraby M, Menshawy M, Dawood N, Hashem $\mathrm{H}$. Predictors of stroke outcome: the role of hemorheology, natural anticoagulants, and serum albumin. Egypt J Neurol Psychiatr Neurosurg. 2018:54(1):18. https://doi.org/10.1186/s41983-018-0019-x.

13. Shimizu K, Shimomura K, Tokuyama Y, Sakurai K, Isahaya K, Takaishi S, et al. Association between inflammatory biomarkers and progression of intracranial large artery stenosis after ischemic stroke. J Stroke Cerebrovasc Dis. 2013;22(3):211-7.

14. Cherubini A, Ruggiero C, Polidori MC, et al. Potential markers of oxidative stress in stroke. Free Radic Biol Med. 2005;39(7):841-52.

15. Qi AQ, Li Y, Liu Q, Si JZ, Tang XM, Zhang ZQ, Qi OD, Chen WB. Thioredoxin is a novel diagnostic and prognostic marker in patients with ischemic stroke. Free Radic Biol Med. 2015;80:129-35.

16. Takagi Y, Mitsui A, Nishiyama A, et al. Overexpression of thioredoxin in transgenic mice attenuates focal ischemic brain damage. Proc Natl Acad Sci U S A. 1999;96(7):4131-6.

17. Wu MH, Song FY, Wei LP, Meng ZY, Zhang ZQ, Qi QD. Serum levels of thioredoxin are associated with stroke risk, severity, and lesion volumes. Mol Neurobiol. 2016;53:677-85

18. Yu T, Zhang W, Lin Y, Li Q, Xue J, Cai Z, Cheng Y, Shao B. Prognostic value of serum thioredoxin levels in ischemic stroke. Neurol Res. 2017. https://doi. org/10.1080/01616412.2017.1359882.

19. Ozkul A, Akyol A, Yenisey C, Arpaci E, Kiylioglu N, Tataroglu C. Oxidative stress in acute ischemic stroke. J. Clin.Neurosci. 2007:14(11):1062-6.

20. Carbonell T, Rama R. Iron, oxidative stress and early neurological deterioration in ischemic stroke. Curr Med Chem. 2007;14(8):857-74

21. Al-Hussain F, lqbal M, Al-Quwayee $M$, Jurays $A B, A l-W a b e l ~ M$, Dayes $S$, et al. The role of serum levels of thioredoxin and thioredoxin-interacting protein in stroke. J Nat Sci Med. 2018;1:55-8.

22. Yusuf S, Hawken S, Ounpuu S, Dans T, Avezum A, Lanas F, McQueen M, Budaj A, Pais P, Varigos J, Lisheng L. Effect of potentially modifiable risk factors associated with myocardial infarction in 52 countries (the INTERHEART Study): case control study. Lancet. 2004:364:937-52.

23. Rodrigo R, Gonzalez J, Paoletto F. The role of oxidative stress in the pathophysiology of hypertension. Hypertension Res. 2011;34:431-40. https://doi. org/10.1038/hr.2010.264

24. Paravicini TM, Touyz RM. Redox signalling in hypertension. Cardiovasc Res. 2006;71:247-58.

25. Selvin E, Erlinger TP. Prevalence of and risk factors for peripheral arterial disease in the United States: results from National Health and Nutrition Examination Survey, 1999-2000. Circulation. 2004;110:738-43 PMID: 15262830.

26. Zalba G, Beloqui O, San José G, Moreno UM, Fortuño A, Díez J. NADPH oxidase-dependent superoxide production is associated with carotid intimamedia thickness in subjects free of clinical atherosclerotic disease. Arterioscler Thromb Vasc Biol. 2005;25:1452-7 PMID: 15860744.

27. Witztum JL, Berliner JA. Oxidized phospholipids and isoprostanes in atherosclerosis. Curr Opin Lipidol. 1998;9:441-8. https://doi.org/10.1097/ 00041433-199810000-00008.

28. Leopold JA, Loscalzo J. Oxidative risk for atherothrombotic cardiovascular disease. Free Radic Biol Med. 2009;47:1673-706. https://doi.org/10.1016/j.free radbiomed.2009.09.009.

29. Sinha K, Das J, Pal PB, Sil PC. Oxidative stress: the mitochondria-dependent and mitochondria-independent pathways of apoptosis. Arch Toxicol. 2013; 87:1157-80. https://doi.org/10.1007/s00204-013-1034-4

30. Taniyama $Y$, Griendling KK. Reactive oxygen species in the vasculature: molecular and cellular mechanisms. Hypertension. 2003;42:1075-81 PMID: 14581295.

31. Forstermann U. Nitric oxide and oxidative stress in vascular disease. Pflugers Arch. 2010;459:923-39 PMID: 20306272.

32. Nanetti $L$, Raffaelli F, Vignini A, Perozzi C. silvestrini M, Bartolini M, Provinciali $L$ and mazzanti $L$. Oxidative stress in ischaemic stroke. Eur J Clin Invest. 2011:41(12):1318-22.

33. Lipton SA. Redox sensitivity of NMDA receptors. Methods Mol Biol. 1999; 128:121-30

\section{Publisher's Note}

Springer Nature remains neutral with regard to jurisdictional claims in published maps and institutional affiliations. 